\title{
DIFFERENTIAL AND INTEGRAL INVARIANTS OF PLANE CURVES AND HORN ANGLES ${ }^{1}$
}

\author{
J. M. FELD
}

1. Introduction. A horn angle has been defined by E. Kasner as a figure formed by an ordered pair of oriented analytic arcs which pass through a common point in a common direction and which have contact of order $n \geqq 1$ at the common point. The order of contact is said to be the order of the horn angle. Thus, in a horn angle of the first order the arcs forming its sides have, at the vertex of the angle, the same inclination (direction) but different curvatures. Horn angles of the first order have a unique absolute conformal invariant, first found by Kasner. ${ }^{2}$ Two relative conformal invariants of horn angles of the first order were given later in a joint paper by Kasner and Comenetz. ${ }^{3}$ Kasner has also determined conformal invariants of the fifth order for horn angles of second order contact. ${ }^{4}$

Kasner and Comenetz obtained the above-mentioned invariants by the use of power series and by repeated differentiation of the CauchyRiemann equations. It is shown in $\$ 2$ of this paper that the relative and absolute conformal invariants of horn angles of the first order can be obtained directly from two standard formulas; one of these expresses $d^{2} y / d x^{2}$ in terms of $u$ and $v$ where $x=x(u)$ and $y=y(v)$; the other expresses the Schwarzian derivative

$$
\{y, x\}=\frac{d^{3} y}{d x^{3}} / \frac{d y}{d x}-\frac{3}{2}\left(\frac{d^{2} y}{d x^{2}} / \frac{d y}{d x}\right)^{2}
$$

in terms of $u$ and $v$ under the same conditions. As a consequence, the invariants are presented in a form which permits of their being interpreted as invariants, not only under the conformal group, but also under a group of multiply differentiable transformations of the type: $x=x(u), y=y(v)$, where $x$ is a function of $u$ alone, and $y$ is a function of $v$ alone. ${ }^{5}$

\footnotetext{
1 Presented to the Society, February 25, 1939.

2 E. Kasner, Conformal geometry, Proceedings of the Fifth International Congress of Mathematicians, Cambridge, 1912, vol. 2, p. 81.

3 E. Kasner and G. Comenetz, Conformal geometry of horn angles, National Academy of Sciences, vol. 22 (1936), p. 303. See also Comenetz, Kasner's invariant and trihornometry, American Mathematical Monthly, vol. 45 (1938), p. 28.

${ }^{4}$ Kasner, Transactions of this Society, vol. 44 (1938), p. 25.

5 Transformations of this type occur, for example, in the papers: Kasner, Transactions of this Society, vol. 16 (1915), pp. 333-349; Cayley, Collected Works, vol. 11, p. 148.
} 
In $\S 3$ we obtain, in terms of what we define as higher Schwarzian derivatives, an infinitude of differential and integral invariants under the inversive group. The fundamental differential inversive invariant, which is of the fifth order, was first obtained by G. Mullins ${ }^{6}$ and later, using other methods, by Liebmann, Kubota, Morley, Patterson and Kasner. ${ }^{7}$ The use of higher Schwarzian derivatives enables us to obtain simply and in compact form an infinitude of relative and absolute differential and integral invariants of curves.

In $\$ 4$ we derive inversive invariants of horn angles of order $n \geqq 3$.

2. Conformal invariants of horn angles of the first order. Let the transformation

$\mathrm{T}$ :

$$
z=\phi(u), \quad \bar{z}=\bar{\phi}(\bar{u}), \quad z=x+i y, \quad \bar{z}=x-i y
$$

map a region $r$ of the gaussian z-plane conformally on the region $r^{\prime}$ of the $u$-plane. Let the analytic arcs ${ }^{8} c_{1}$ and $c_{2}$ in the region $r$ be the first and second sides respectively of a horn angle of the first order, $\alpha$, the vertex of which is at the point $P$. Let $T$ map $c_{1}$ and $c_{2}$ upon the arcs $c_{1}^{\prime}$ and $c_{2}^{\prime}$ respectively in $r^{\prime}$ where the latter form a horn angle of the first order, $\alpha^{\prime}$, with vertex at $P^{\prime}$. Let $\theta$ represent the common angle of inclination at $P$ of $c_{1}$ and $c_{2}, k_{1}$ and $k_{2}$ their respective curvatures at $P$, while $d k_{1} / d s_{1}$ and $d k_{2} / d s_{2}$ represent respectively the values at $P$ of the derivative of curvature with respect to arc-length. The same symbols primed represent the corresponding quantities associated with $c_{1}^{\prime}$ and $c_{2}^{\prime}$ at $P^{\prime}$.

Let $f(z, \bar{z})=0$ be the self-conjugate equation (in isotropic coordinates) of a curve $c$ in the $z$-plane. Likewise, let arcs $c_{1}$ and $c_{2}$ be represented by $f_{1}(z, \bar{z})=0, f_{2}(z, \bar{z})=0$, respectively, and let their images $c^{\prime}, c_{1}^{\prime}, c_{2}^{\prime}$ on the $u$-plane be given by $g(u, \bar{u})=0, g_{1}=0, g_{2}=0$, respectively. By $d^{n} z / d \bar{z}^{n}, d^{n} z_{1} / d \bar{z}_{1}^{n}, d^{n} z_{2} / d \bar{z}_{2}^{n}$ we designate the $n$th derivatives taken along $c, c_{1}$ and $c_{2}$ respectively. Likewise $d^{n} u / d \bar{u}^{n}, d^{n} u_{1} / d \bar{u}_{1}^{n}$ and $d^{n} u_{2} / d \bar{u}_{2}^{n}$ represent respectively derivatives along $c^{\prime}, c_{1}^{\prime}$ and $c_{2}^{\prime}$ in the $u$-plane.

Since

${ }^{6}$ Columbia University Dissertation, 1917.

${ }^{7} \mathrm{H}$. Liebmann, Beiträge zur Inversionsgeometrie der Kurven, Sitzungsberichte der Bayerischen Akademie der Wissenschaften, Munich, vol. 1 (1923), p. 79. T. Kubota, Beiträge zur Inversionsgeometrie, Tôhoku Imperial University Science Reports, vol. 13 (1924-1925), p. 243. F. Morley, On differential inversive geometry, American Journal of Mathematics, vol. 46 (1926), p. 144. B. Patterson, The differential invariants of inversive geometry, ibid., vol. 50 (1928), p. 553. E. Kasner, Transactions of this Society, vol. 44 (1938), p. 25.

${ }^{8}$ This restriction can be relaxed to require only that the arcs possess derivatives of the third order. 


$$
\frac{d z}{d \bar{z}}=\left(\frac{d z}{d u} / \frac{d \bar{z}}{d \bar{u}}\right) \frac{d u}{d \bar{u}}
$$

we obtain

$$
\begin{array}{r}
\frac{d^{2} z}{d \bar{z}^{2}}=\left(1 / \frac{d \bar{z}}{d \bar{u}}\right)\left[\left(\frac{d z}{d u} / \frac{d \bar{z}}{d \bar{u}}\right) \frac{d^{2} u}{d \bar{u}^{2}}\right. \\
\left.+\left(\frac{d u}{d \bar{u}}\right)^{2} \frac{d}{d u}\left(\frac{d z}{d u} / \frac{d \bar{z}}{d \bar{u}}\right)\right] .
\end{array}
$$

Evidently the derivatives of $z$ with respect to $u$ as well as of $\bar{z}$ with respect to $\bar{u}$ are independent of the particular curve $c$; furthermore, $\alpha^{\prime}$ being a horn angle of the first order, $d u_{1} / d \bar{u}_{1}=d u_{2} / d \bar{u}_{2}$ at $P^{\prime}$; consequently, by applying equation (2.2) to $c_{i}$ and $c_{i}^{\prime}$, and subtracting the results, we get

$$
\frac{d^{2} z_{2}}{d \bar{z}_{2}^{2}}-\frac{d^{2} z_{1}}{d \bar{z}_{1}^{2}}=\left[\frac{d z}{d u} /\left(\frac{d \bar{z}}{d \bar{u}}\right)^{2}\right]\left[\frac{d^{2} u_{2}}{d \bar{u}_{2}^{2}}-\frac{d^{2} u_{1}}{d \bar{u}_{1}^{2}}\right] .
$$

Since $d z_{1} / d \bar{z}_{1}=d z_{2} / d \bar{z}_{2}$ at $P$, division of equation (2.3) by equation (2.1) applied to $c_{i}$ and $c_{i}^{\prime}$ results in

$$
\begin{aligned}
\frac{d^{2} z_{2}}{d \bar{z}_{2}^{2}} / \frac{d z_{2}}{d \bar{z}_{2}}-\frac{d^{2} z_{1}}{d \bar{z}_{1}^{2}} / \frac{d z_{1}}{d \bar{z}_{1}} & \\
= & \left(1 / \frac{d \bar{z}}{d \bar{u}}\right)\left[\frac{d^{2} u_{2}}{d \bar{u}_{2}^{2}} / \frac{d u_{2}}{d \bar{u}_{2}}-\frac{d^{2} u_{1}}{d \bar{u}_{1}^{2}} / \frac{d u_{1}}{d \bar{u}_{1}}\right] .
\end{aligned}
$$

Consequently, the left members of (2.3) and (2.4) represent complex relative conformal invariants of the second order of horn angles of the first order.

When the variables in the Schwarzian derivative $\{y, x\}$ undergo a transformation $x=x(s), y=y(t)$, it was proved by Cayley ${ }^{9}$ that

$$
\{y, x\}=\left(\frac{d t}{d x}\right)^{2}\{y, t\}-\left(\frac{d s}{d x}\right)^{2}\{x, s\}+\left(\frac{d s}{d x}\right)^{2}\{t, s\} .
$$

In isotropic coordinates this becomes

$$
\{z, \bar{z}\}=\left(1 / \frac{d \bar{z}}{d \bar{u}}\right)^{2}\left[\{u, \bar{u}\}-\{\bar{z}, \bar{u}\}+\{z, u\}\left(\frac{d u}{d \bar{u}}\right)^{2}\right]
$$

showing the effect of a direct conformal transformation on $\{z, \bar{z}\}$.

${ }^{\ominus}$ A. Cayley, "On the Schwarzian derivative and polyhedral functions," Collected Works, vol. 11, p. 148. 
From $\left(2.5^{\prime}\right)$ we find that, for the horn angles $\alpha$ and $\alpha^{\prime}$ at points $P$ and $P^{\prime}$,

$$
\left\{z_{2}, \bar{z}_{2}\right\}-\left\{z_{1}, \bar{z}_{1}\right\}=\left(1 / \frac{d \bar{z}}{d \bar{u}}\right)^{2}\left[\left\{u_{2}, \bar{u}_{2}\right\}-\left\{u_{1}, \bar{u}_{1}\right\}\right] .
$$

Consequently, horn angles of the first order possess the complex relative conformal invariant of the third order given by the left member of (2.6).

From the relative invariants (2.4) and (2.6) of index one and two respectively we obtain the absolute invariant of the third order

$$
M_{12}=\frac{\left(\frac{d^{2} z_{2}}{d \bar{z}_{2}^{2}} / \frac{d z_{2}}{d \bar{z}_{2}}-\frac{d^{2} z_{1}}{d \bar{z}_{1}^{2}} / \frac{d z_{1}}{d \bar{z}_{1}}\right)^{2}}{\left\{z_{2}, \bar{z}_{2}\right\}-\left\{z_{1}, \bar{z}_{1}\right\}} .
$$

A simple computation indicates that

$$
\begin{aligned}
\frac{d z}{d \bar{z}} & =e^{2 i \theta}, \quad \frac{d^{2} z}{d \bar{z}^{2}}=2 i e^{3 i \theta} k, \\
\frac{d^{3} z}{d \bar{z}^{3}} & =2 i e^{4 i \theta}\left(\frac{d k}{d s}+3 i k^{2}\right) .
\end{aligned}
$$

Substitution of the values given by (2.7) in (2.4) and (2.6) shows that each of these complex relative invariants implies one real relative invariant, namely,

$$
k_{2}-k_{1} \text { and } d k_{2} / d s_{2}-d k_{1} / d s_{1}
$$

respectively. In this form they were given in a joint paper by Kasner and Comenetz. ${ }^{10}$

Substitution from (2.7) in $M_{12}$ leads us likewise to a single real invariant, namely, Kasner's unique absolute conformal invariant of the third order of horn angles of the first order:

$$
M_{12}^{*}=\frac{\left(k_{2}-k_{1}\right)^{2}}{d k_{2} / d s_{2}-d k_{1} / s_{1}}, \quad M_{12}=2 i M_{12}^{*} .
$$

Comenet $\mathrm{z}^{11}$ has proved that if a conformal transformation, $S$ :

$$
x_{2}=\phi\left(x_{1}, y_{1}\right), \quad y_{2}=\psi\left(x_{1}, y_{1}\right)
$$

maps a region $r_{1}$ in the $x_{1} y_{1}$-plane on a region $r_{2}$ in the $x_{2} y_{2}$-plane, so

${ }^{10}$ Kasner and Comenetz, National Academy of Sciences, loc. cit.

${ }^{11}$ American Mathematical Monthly, vol. 45 (1938), p. 86. 
that a curve $c_{1}$ in $r_{1}$ is mapped on $c_{2}$ in $r_{2}$, then

$$
k_{2}=m k_{1}+m_{y_{1}} \cos \theta_{1}-m_{x_{1}} \sin \theta_{1}
$$

and

$$
d k_{2} / d s_{2}=m^{2} d k_{1} / d s_{1}+m\left[m_{x_{1} y_{1}} \cos 2 \theta_{1}+\frac{1}{2}\left(m_{y_{1} y_{1}}-m_{x_{1} x_{1}}\right) \sin 2 \theta_{1}\right],
$$

where $m=1 /(J)^{1 / 2}$ and $J$ is the jacobian of $S$. These formulas, describing the laws of transformation of curvature and rate of change of curvature under conformal mapping, are equivalent, except for factors, to (2.2) and $\left(2.5^{\prime}\right)$ respectively. Consequently, Comenetz's formulas are obtainable by appropriately interpreting standard identities that express the second derivative and Schwarzian derivative in terms of new variables. This remark enables us to regard (2.4), (2.6) and $M_{12}$ as invariants of horn angles under a group other than the conformal group.

Explicitly, let the curves $f_{1}(x, y)=0, f_{2}(x, y)=0$ form a horn angle of the first order in a region $R$ of the $x y$-plane, $x, y$ being cartesian coordinates. Let the transformation $K: x=x(s), y=y(t)$, map $R$ uniformly and continuously on a region $R^{\prime}$ of the st-plane. We assume that $K$ is provided with at least third derivatives in $R$. Then $K$ maps the horn angle in $R$ on a horn angle of the first order in $R^{\prime}$. If we now write equations (2.1) and (2.2) in terms of $x, y$ and $s, t$, thereby expressing the transformations that $d y / d x$ and $d^{2} y / d x^{2}$ undergo under $K$, just as (2.5) does for $\{y, x\}$, we obtain the result that horn angles of the first order, under a group of transformations of type $K$, have the relative ${ }^{12}$ invariants

$$
A_{12}=\frac{d^{2} y_{2}}{d x_{2}^{2}} / \frac{d y_{2}}{d x_{2}}-\frac{d^{2} y_{1}}{d x_{1}^{2}} / \frac{d y_{1}}{d x_{1}}, \quad B_{12}=\left\{y_{2}, x_{2}\right\}-\left\{y_{1}, x_{1}\right\}
$$

and the absolute invariant $A_{12}^{2} / B_{12}$.

3. Higher Schwarzian derivatives and inversive invariants of curves. The inversive group in the gaussian plane is given by

$$
z=(a u+b) /(c u+d), \quad z=\bar{u}, \quad a d-b c \neq 0 .
$$

The first of these equations represents a subgroup $G$, containing only directly conformal inversive transformations. We first consider the transformations of $G$. Let any member of $G$ transform the curve $C$ :

12 The term relative invariants as used here and elsewhere in this paper, means a quantity that is multiplied by some function which depends only upon the transformation; however, contrary to usual practice, the multiplier need not be a power of the jacobian of the transformation. 
$f(z, \bar{z})=0$ into $C^{\prime}: g(u, \bar{u})=0$. Then, inasmuch as for such transformations $\{z, u\}=\{\bar{z}, \bar{u}\}=0,\left(2.5^{\prime}\right)$ reduces to

$$
\{z, \bar{z}\}=\left(1 / \frac{d \bar{z}}{d \bar{u}}\right)^{2}\{u, \bar{u}\}
$$

from which we gather that $\{z, \bar{z}\}$ is a relative invariant of curves un$\operatorname{der} G$. Noting that $d \bar{z}=(d \bar{z} / d \bar{u}) d \bar{u}$, we obtain

$$
\{z, \bar{z}\} d \bar{z}^{2}=\{u, \bar{u}\} d \bar{u}^{2}
$$

indicating that $\{z, \bar{z}\} d \bar{z}^{2}$ is an absolute invariant under $G$. Since

$$
\{z, \bar{z}\}=2 i e^{2 i \theta} \frac{d k}{d s}, \quad d \bar{z}=e^{-i \theta} d s
$$

we get the real absolute invariant

$$
(-1 / 2 i)\{z, \bar{z}\} d \bar{z}^{2}=-d k d s .
$$

If we let $d \lambda^{2}= \pm d k d s$, choosing the sign that makes $d \lambda^{2}$ positive, the integral invariant $\lambda$ represents what has come to be known as the inversive arc-length. ${ }^{13}$ Letting $\rho$ represent radius of curvature,

$$
-d k d s=d \rho d s / \rho^{2},
$$

under which form this invariant was found, by other means, by Liebmann and Kubota.

Differentiating (3.1) twice, we obtain

$$
\frac{d}{d \bar{z}}\{z, \bar{z}\}=\left(1 / \frac{d \bar{z}}{d \bar{u}}\right)^{3} \frac{d}{d \bar{u}}\{u, \bar{u}\}-2\{u, \bar{u}\} \frac{d^{2} \bar{z}}{d \bar{u}^{2}} /\left(\frac{d \bar{z}}{d \bar{u}}\right)^{4}
$$

and

$$
\begin{aligned}
\frac{d^{2}}{d \bar{z}^{2}}\{z, \bar{z}\}= & \left(1 / \frac{d \bar{z}}{d \bar{u}}\right)^{4} \frac{d^{2}}{d \bar{u}^{2}}\{u, \bar{u}\} \\
& -5 \frac{d}{d \bar{u}}\{u, \bar{u}\} \frac{d^{2} \bar{z}}{d \bar{u}^{2}} /\left(\frac{d \bar{z}}{d \bar{u}}\right)^{5} \\
& -2\{u, \bar{u}\}\left[\frac{d^{3} \bar{z}}{d \bar{u}^{3}} /\left(\frac{d \bar{z}}{d \bar{u}}\right)^{5}\right. \\
& \left.-4\left(\frac{d^{2} \bar{z}}{d \bar{u}^{2}}\right)^{2} /\left(\frac{d \bar{z}}{d \bar{u}}\right)^{6}\right] .
\end{aligned}
$$

${ }^{13}$ See, e.g., Patterson, American Journal of Mathematics, loc. cit. 
From (3.1), (3.2) and (3.3) we then obtain, recalling that $\{z, u\}=0$,

$$
\begin{aligned}
\frac{d^{2}}{d \bar{z}^{2}}\{z, \bar{z}\} /\{z, \bar{z}\} & -\frac{5}{4}\left[\frac{d}{d \bar{z}}\{z, \bar{z}\} /\{z, \bar{z}\}\right]^{2} \\
= & \left(1 / \frac{d \bar{z}}{d \bar{u}}\right)^{2}\left[\frac{d^{2}}{d \bar{u}^{2}}\{u, \bar{u}\} /\{u, \bar{u}\}\right. \\
& \left.-\frac{5}{4}\left(\frac{d}{d \bar{u}}\{u, \bar{u}\} /\{u, \bar{u}\}\right)^{2}\right] .
\end{aligned}
$$

Let the left member of (3.4) be represented by the symbol $\{z, \bar{z}\}_{2}$, which we shall call the second Schwarzian derivative of $z$ with respect to $\bar{z}$. It follows from (3.4) that $\{z, \bar{z}\}_{2}$ is a relative invariant of the fifth order under the direct inversive group. In our new notation (3.4) becomes

$$
\{z, \bar{z}\}_{2}=\left(1 / \frac{d \bar{z}}{d \bar{u}}\right)^{2}\{u, \bar{u}\}_{2} .
$$

Generalizing the definition of the second Schwarzian derivative by a process of recurrence, we shall call

$$
\{z, \bar{z}\}_{n+1}=\frac{d^{2}}{d \bar{z}^{2}}\{z, \bar{z}\}_{n} /\{z, \bar{z}\}_{n}-\frac{5}{4}\left(\frac{d}{d \bar{z}}\{z, \bar{z}\}_{n} /\{z, \bar{z}\}_{n}\right)^{2}
$$

the $(n+1)$-th Schwarzian derivative. We let $\{z, \bar{z}\}_{1} \equiv\{z, \bar{z}\}$.

It can be shown by induction ${ }^{14}$ that the Schwarzian derivatives of order $n \geqq 1$ are all relative invariants of index two under the direct inversive group, or that

$$
\{z, \bar{z}\}_{k}=\left(1 / \frac{d \bar{z}}{d \bar{u}}\right)^{2}\{u, \bar{u}\}_{k}, \quad k \geqq 1 .
$$

Consequently, the ratios

$$
\{z, \bar{z}\}_{j} /\{z, \bar{z}\}_{k}, \quad j \neq k ; j, k=1,2,3, \cdots,
$$

are absolute differentual invariants of plane curves under $G$. Of these, the one of lowest order, namely that of the fifth order, is

$$
I_{5}=\{z, \bar{z}\}_{2} /\{z, \bar{z}\} .
$$

We proceed to express $I_{5}$ in terms of $k$ and derivatives of $k$ with respect to $s$.

${ }^{14}$ The proof of this statement involves straightforward computation, which we shall omit. 
From

$$
\{z, \bar{z}\}=2 i e^{2 i \theta} d k / d s
$$

we obtain

$$
\frac{d}{d \bar{z}}\{z, \bar{z}\}=2 e^{3 i \theta}\left(i \frac{d^{2} k}{d s^{2}}-2 k \frac{d k}{d s}\right)
$$

and

$$
\frac{d^{2}}{d \bar{z}^{2}}\{z, \bar{z}\}=2 e^{4 i \theta}\left[i \frac{d^{3} k}{d s^{3}}-5 k \frac{d^{2} k}{d s^{2}}-6 i k^{2} \frac{d k}{d s}-2\left(\frac{d k}{d s}\right)^{2}\right] .
$$

Substituting these expressions in $\{z, \bar{z}\}_{2}$, we find

$$
\{z, \bar{z}\}_{2}=e^{2 i \theta}\left[\frac{d^{3} k}{d s^{3}} / \frac{d k}{d s}-\frac{5}{4}\left(\frac{d^{2} k}{d s^{2}} / \frac{d k}{d s}\right)^{2}-k^{2}+2 i \frac{d k}{d s}\right]
$$

and therefore

$$
I_{5}=1-\frac{i}{2}\left[\frac{d^{3} k}{d s^{3}} /\left(\frac{d k}{d s}\right)^{2}-\frac{5}{4}\left(\frac{d^{2} k}{d s^{2}}\right)^{2} /\left(\frac{d k}{d s}\right)^{3}-k^{2} / \frac{d k}{d s}\right] .
$$

Hence, $I_{5}$ implies only one real invariant, namely

$$
I_{5}^{*}=\frac{d^{3} k}{d s^{3}} /\left(\frac{d k}{d s}\right)^{2}-\frac{5}{4}\left(\frac{d^{2} k}{d s^{2}}\right)^{2} /\left(\frac{d k}{d s}\right)^{3}-k^{2} / \frac{d k}{d s} .
$$

In terms of $\rho$ and derivatives of $\rho$ with respect to $s$,

$$
\begin{aligned}
I_{5}^{*}=[-4 & \frac{d^{3} \rho}{d s^{3}} \frac{d \rho}{d s} \rho^{2}+4 \frac{d^{2} \rho}{d s^{2}}\left(\frac{d \rho}{d s}\right)^{2} \rho-4\left(\frac{d \rho}{d s}\right)^{4} \\
& \left.+5\left(\rho \frac{d^{2} \rho}{d s^{2}}\right)^{2}+4\left(\frac{d \rho}{d s}\right)^{2}\right] /\left[4\left(\frac{d \rho}{d s}\right)^{3}\right]
\end{aligned}
$$

in which form this invariant was first obtained by G. Mullins. ${ }^{15}$

Recalling that the inversive arc length $\lambda=\int( \pm d k / d s)^{1 / 2} d s$ and that therefore $d k / d s=(d \lambda / d s)^{2}$ we find that $I_{5}^{*}$ assumes the simple form

$$
I_{5}^{*}=\left(2\{\lambda, s\}-k^{2}\right) /\left(\frac{d \lambda}{d s}\right)^{2}
$$

From (3.6) it obviously follows that

$$
\{z, \bar{z}\}_{k} d \bar{z}^{2}=\{u, \bar{u}\}_{k} d \bar{u}^{2}, \quad k \geqq 1 .
$$

${ }^{15}$ Columbia University Dissertation, 1917. 
Consequently,

$$
\lambda_{k}=\int\left(\{z, \bar{z}\}_{k}\right)^{1 / 2} d \bar{z}
$$

is an integral invariant under $G$. However, $\lambda_{k}$, being complex, implies two real invariants, one of which may be only a constant. When $k=1$ the unique non-constant invariant, as shown above, is the inversive arc-length.

Under the complete inversive group, which includes the group $G$ and the involutory transformation $z=\bar{u}$, the absolute values of the invariants referred to above are invariant.

4. Inversive invariants of horn angles. From (3.6) it obviously follows that curvilinear angles, and therefore horn angles of order $n$, $n \geqq 1$, have, under the direct inversive group $G$, the absolute invariants of odd order, namely $2 k+1$ :

$$
\left\{z_{2}, \bar{z}_{2}\right\}_{k} /\left\{z_{1}, \bar{z}_{1}\right\}_{k},
$$$$
k \geqq 1 \text {. }
$$

However, we can obtain from (3.6) certain other, less obvious horn angle invariants. Let $\alpha$ be a horn angle of order $2 n+1, n \geqq 1$. Differentiation of (3.6) results in

$$
\begin{aligned}
\frac{d}{d \bar{z}}\{z, \bar{z}\}_{n}= & \left(1 / \frac{d \bar{z}}{d \bar{u}}\right)^{3} \frac{d}{d \bar{u}}\{u, \bar{u}\}_{n} \\
& -2\{u, \bar{u}\}_{n} \frac{d^{2} \bar{z}}{d \bar{u}^{2}} /\left(\frac{d \bar{z}}{d \bar{u}}\right)^{4} .
\end{aligned}
$$

Since $\alpha$ is of order $2 n+1,\left\{z_{1}, \bar{z}_{1}\right\}_{n}=\left\{z_{2}, \bar{z}_{2}\right\}_{n},\left\{u_{1}, \bar{u}_{1}\right\}_{n}=\left\{u_{2}, \bar{u}_{2}\right\}_{n}$ at the vertex of the horn angle; consequently, by subtracting the equations obtained by stating (4.1) for the two sides of $\alpha$ we find that

$$
\begin{aligned}
\frac{d}{d \bar{z}_{2}}\left\{z_{2}, \bar{z}_{2}\right\}_{n}- & \frac{d}{d \bar{z}_{1}}\left\{z_{1}, \bar{z}_{1}\right\}_{n} \\
& =\left(1 / \frac{d \bar{z}}{d \bar{u}}\right)^{3}\left[\frac{d}{d \bar{u}_{2}}\left\{u_{2}, \bar{u}_{2}\right\}_{n}-\frac{d}{d \bar{u}_{1}}\left\{u_{1}, \bar{u}_{1}\right\}_{n}\right] .
\end{aligned}
$$

Therefore, the left member of (4.2) represents a relative inversive invariant of order $2 n+2$ and index three of horn angles of order $2 n+1$, $n \geqq 1$.

Let $\alpha$ be a horn angle of even order: $2 n+2, n \geqq 1$. Now it follows from (3.6), by subtraction of (3.5) stated for the two sides of $\alpha$ that 


$$
\begin{aligned}
\frac{d^{2}}{d \bar{z}_{2}^{2}}\left\{z_{2}, \bar{z}_{2}\right\}_{n} & -\frac{d^{2}}{d \bar{z}_{1}^{2}}\left\{z_{1}, \bar{z}_{1}\right\}_{n} \\
& =\left(1 / \frac{d \bar{z}}{d \bar{u}}\right)^{4}\left[\frac{d^{2}}{d \bar{u}_{2}^{2}}\left\{u_{2}, \bar{u}_{2}\right\}_{n}-\frac{d^{2}}{d \bar{u}_{1}^{2}}\left\{u_{1}, \bar{u}_{1}\right\}_{n}\right] .
\end{aligned}
$$

Consequently, horn angles of order $2 n+2, n \geqq 1$, have, under the direct inversive group, the relative invariant of order $2 n+3$ and index four given by (4.3).

Brooklyn College 